\title{
A study of Specialized Resource Function mapping alternatives for Integrated IN/B- ISDN architectures
}

\author{
G. T. Kolyvas, S. E. Polykalas, I. S. Venieris \\ Electrical \& Computer Engineering Department \\ National Technical University of Athens \\ 9 Heroon Polytechniou Str., 15773 Zographou, Athens, Greece \\ Phone (30) 1-772 2551 Fax. (30) 1- 7722534 \\ e_mail: ivenieri@cc.ece.ntua.gr
}

\begin{abstract}
The introduction of Intelligent Network (IN) functionality to broadband networks with conventional signalling systems allows the fast and future safe provision of advanced multimedia services. Since novel services will require intense interactions with the network resulting in the exchange of a high number of control messages, the distribution of IN functional entities to network elements becomes a dominant factor in the overall system performance. In this paper we analyze the functions required for an integrated IN/B-ISDN architecture and develop a set of models for representing protocols and IN functional entities. Our purpose is to identify the system bottlenecks and to evaluate alternatives for functional to physical entity mapping that allow the most efficient operation of the system. Emphasis is given to the Specialized Resource Function entity location.
\end{abstract}

Keywords

Intelligent Networks, Intelligent Peripheral, functionality mapping, performance evaluation 


\section{INTRODUCTION}

The Intelligent Network (IN) concept provides a flexible and modular methodology for the description and specification design and implementation of complex multimedia services. Services are created by establishing relations among Service Independent Functions in a manner that guarantees a high degree of transparency with respect to both the service implementation and the underlying physical network infrastructure. This flexibility allows network operators to upgrade their systems easily and in a cost effective fashion by taking advantage of the ability to allocate IN functionality and resources within the network without restrictions.

A tentative task in the design of an IN-based architecture is the distribution of IN functions to physical entities. In principle a physical entity is a functional group which can include service, interconnect and communications functions. A physical entity may provide the entire set of functions or a subset of them. IN functions inside a physical entity are organised into functional entities. A functional entity can be present in more than one physical entities but it cannot be split among multiple physical entities.

In this paper we investigate alternatives for the mapping of IN functional to physical entities for the case of an integrated IN/B-ISDN architecture (Hussmann, 1995), (Wu, 1995). The IN functional entities that participate in the exchange and process of the control information related to the request and provision of an IN service are the Call Control Function (CCF), the Service Switching Function (SSF) which identifies IN calls and triggers the service logic in the Service Control Function (SCF), the Specialized Resource Function (SRF) which manages the communication between the user and the IN, and the Service Data Function (SDF) containing service and call related data. The physical entities of the IN/B-ISDN network are the Broadband Service Switching Point (B-SSP) which is a typical ATM switch enhanced with the ability to recognize IN service requests and to communicate with IN entities and the B-Service Control Point (B-SCP) which provides the IN service related functionality. While CCF and SSF are always located in the B-SSP and SCF in the B-SCP, the SRF can be found either in an integrated B-SSP or in a separate B-Intelligent Peripheral (B-IP). Similarly the SDF can be located either in the B-SCP or in a separate B-Service Data Point (BSDP). The location of these functional entities is an important issue with an impact on the architecture, topology and scalability of the IN/B-ISDN system.

Apart from the IN functional entities, the physical entities should also contain appropriate signalling protocols enabling the establishment and release of bearer connections for IN service calls. In the IN/B-ISDN system under study, the signalling protocols are those currently standardized for B-ISDN, that is the Q.2931 protocol in the UNI (ITU-T, Q.2931) and the B-ISUP (B-ISDN User Part) in the NNI (ITU-T, Q.2761). 
The purpose of this paper is to provide performance driven guidelines for the design of IN based broadband networks. Similar work has been performed in the framework of Signalling System No 7 (SS7) and B-ISDN signalling protocols (Bafutto, 1994), (Lazar, 1994), (Veeraraghavan, 1995), (Veeraraghavan), (Willmann, 1990), (Xou, 1994), (La Porta, 1993) notwithstanding that in our case the integrated service facilities and the employment of IN capabilities necessitate more complex user-to-network and network-to-network transaction models (Hussmann, 1995). Our study undertakes issues related to the scalability of the IN broadband network by investigating and comparing alternatives for the mapping of SRF to physical entities. Since our emphasis is mainly placed on the quantitative than the qualitative analysis, we develop a set of models for evaluating the performance of the system in terms of the maximum number of admitted calls and the call set up delay. Despite the many alternatives for distributing functional entities to physical entities, the IN communication protocols (i.e. B-Intelligent Network Application Part - B-INAP (ITU-T, Q.1218)) and the functionality of the functional entities are almost stable or at least on their way of standardization. Therefore modeling of the functional entities can be performed independently of their location and furthermore the physical entity model can be derived as a combination of the standalone functional entities models.

The paper is organized as follows: In Section 2 we present the alternatives for the mapping of functional to physical entities and the resulting network architectures of an IN-based B-ISDN which are evaluated in the rest of the paper. Section 3 includes a set of detailed models developed to accurately describe functional entities and signalling protocols. The interaction among physical entities as well as the sequence of events in the functional entities are defined in Section 4 for a typical Broadband Video on Demand (B-VoD) call establishment and release. The performance results of Section 5 are discussed and evaluated giving input to a number of conclusions regarding system design options summarized in Section 6.

\section{MAPPING OF IN FUNCTIONAL ENTITIES TO NETWORK PHYSICAL ENTITIES}

The generic network configuration model of the IN-based B-ISDN signalling system contains all those physical entities that contribute to the establishment and provision of an IN service (ITU-T, Q.1214). These entities are the user terminal (e.g. a set top box or an ATM PC), the B-SSP, the B-SCP and the service provider which in the case of an interactive B-VoD service is the Video Server (VS). The BSSP can be an integrated B-SSP in which case it includes not only the CCF and SSF functional entities but also the SRF, or a simple B-SSP. In the latter case a BIP is required to provide the necessary specialized resources to the user. The emerging alternatives for the generic network configuration with respect to the location of the SRF functional entity are illustrated in Figure 1. In alternative A the path between the user terminal and the VS is shown to involve an integrated B-SSP, 


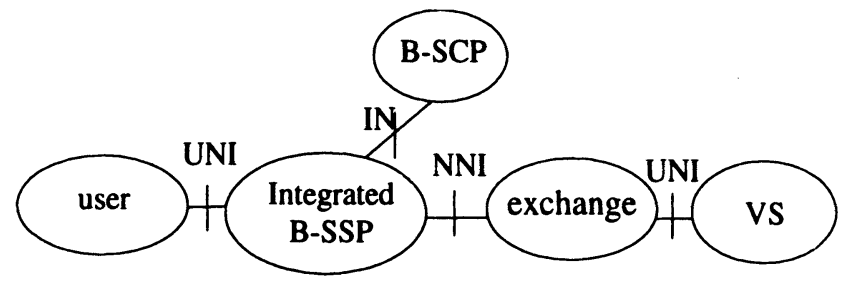

Topology A with integrated B-SSP

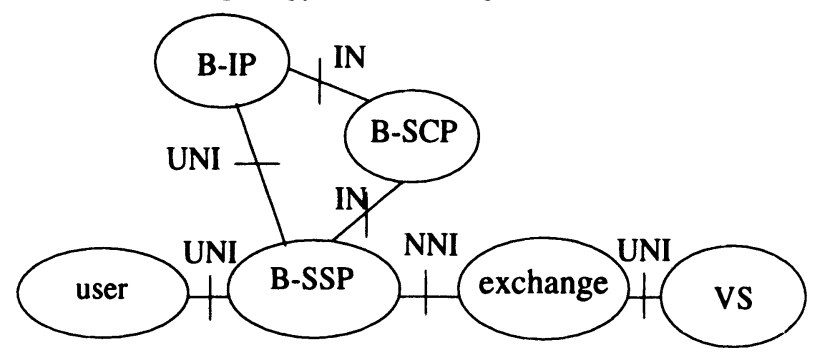

Topology B with separate B-IP

Mapping of Functional Entities to Physical Entities

Figure 1 Alternatives for an IN-based B-ISDN network configuration
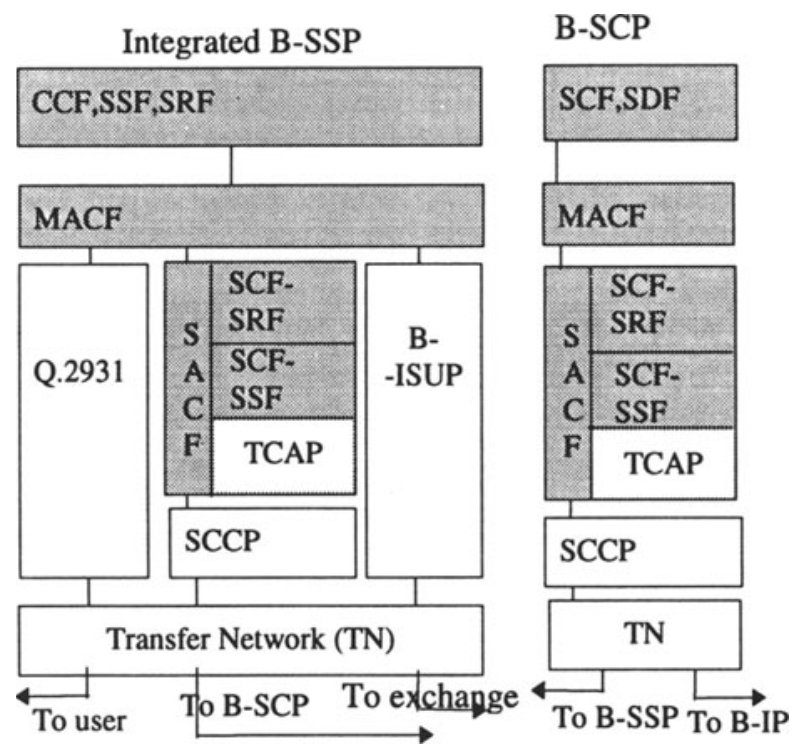

Figure 2 Protocol stacks for alternative A.

a B-SCP and an exchange. In alternative B, the B-SSP is not integrated however a B-IP is now present. The interface between the user terminal and the B-SSP is a 
B-IP

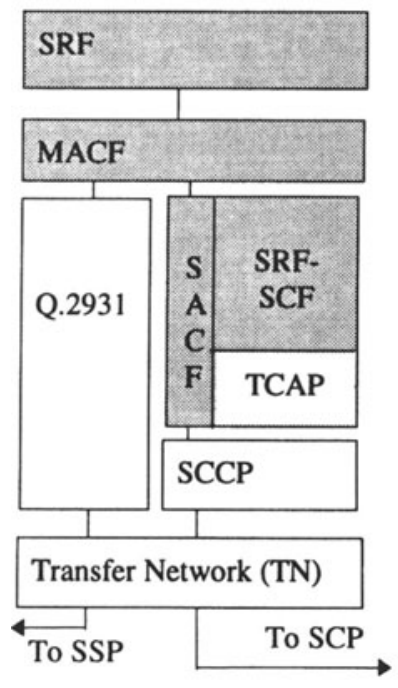

\section{B-SCP}

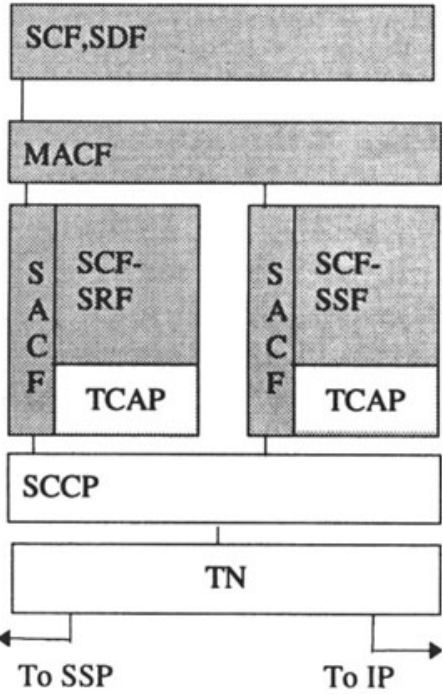

B-SSP

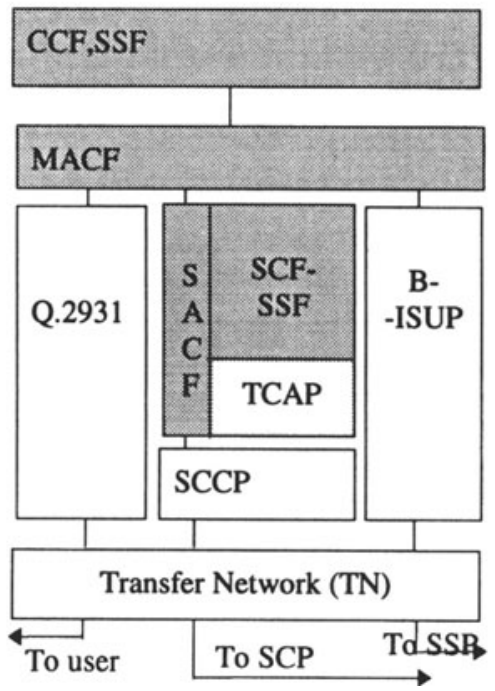

Figure 3 Protocol stacks for alternative B.

broadband UNI with Q.2931, Signalling ATM Adaptation Layer (S-AAL) (ITU-T, Q.1200), and ATM protocols and between the B-SSP and the exchange a broadband NNI with B-ISUP and Message Transfer Part 3 (MTP-3) (ITU-T, Q.701) over S-AAL protocols . The B-SCP is connected to the B-SSP through an IN interface supporting B-INAP operations (ITU-T, Q.1218) at the upper level and 
Transaction Capability Application Part (TCAP) (ITU-T, Q.771) and Signalling Connection Control Part (SCCP) protocols (ITU-T, Q.711) at the lower level lying over the transfer network. In alternative $B$ the user is connected to the B-IP through the B-SSP with a UNI protocol and the B-IP to the B-SCP through an IN interface with the same stack as the one of the B-SCP. The detailed protocol stacks of the physical entities are illustrated in Figures 2 and 3 for alternatives A and B respectively.

\section{FUNCTIONAL ENTITY AND SIGNALLING PROTOCOL MODELS}
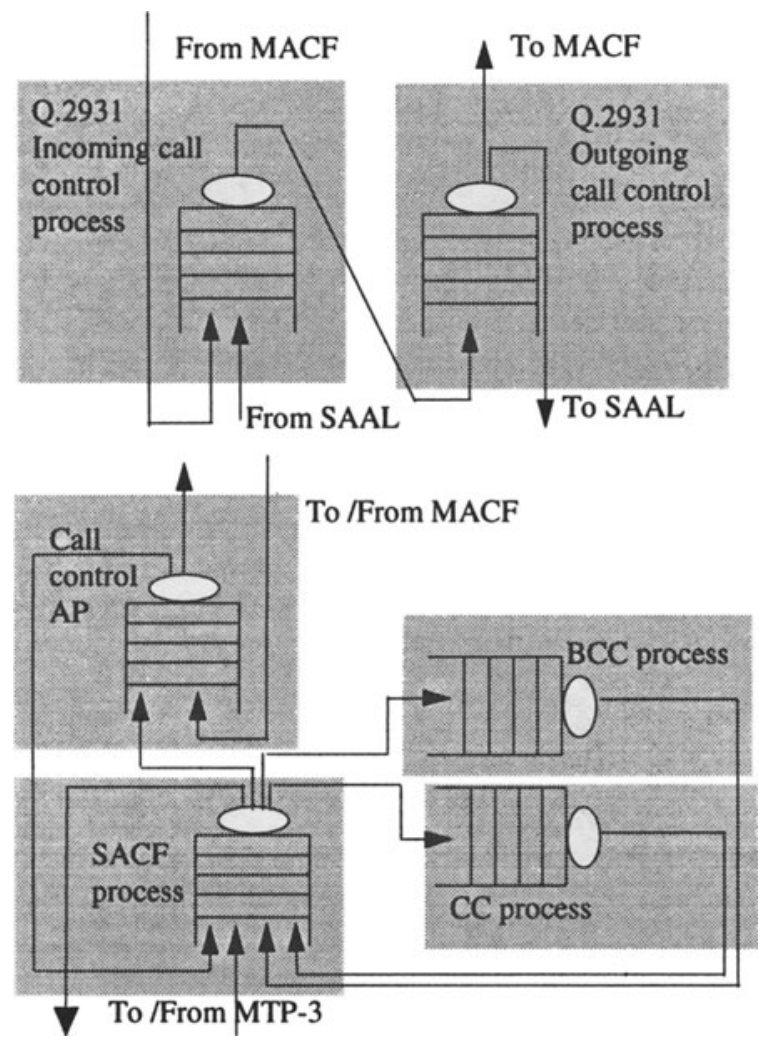

Figure 4 Q.2931/B-ISUP Models.

Since the focus of this paper is on the upper signalling and IN protocol layers, the transfer network is not modeled in detail; i.e., only the protocol overhead added in each layer is taken into account to determine the number of cells a signalling message is segmented into, while for each of the functional entities and the upper signalling 
protocols that are involved in a call set up and release phase of an IN service, a model is developed. The model includes the protocol layer functions, as well as the information flow among protocols and the protocol internal functions. The servers of each queue illustrated in Figures 4-7 represent the processes activated in each functional entity or protocol. We assume that a single processor serves the entire functional entity or protocol and that time sharing among processes is exercised by a scheme of priorities.

Q.2931 Model. The Q.2931 model is illustrated in Figure 4. The call/connection processing control has been divided in two processes, the incoming and outgoing call/connection process.

B-ISUP model. On account of the fact that in our study we focus on the IN functionality and performance, we have assumed that IN and signalling messages pass transparently over the network transfer part. Therefore we are not interested in compatibility and maintenance functions of the B-ISUP but only in the call and bearer connection control functions. So, the model of Figure 4 consists of the BCC, CC ASEs, the SACF and the call control AP. When the SACF receives a message, it distributes information to ASEs according to the protocol rules. The output from the ASEs is received by the SACF which forwards an appropriate message to the application process or to the underlying MTP-3 protocol layer.

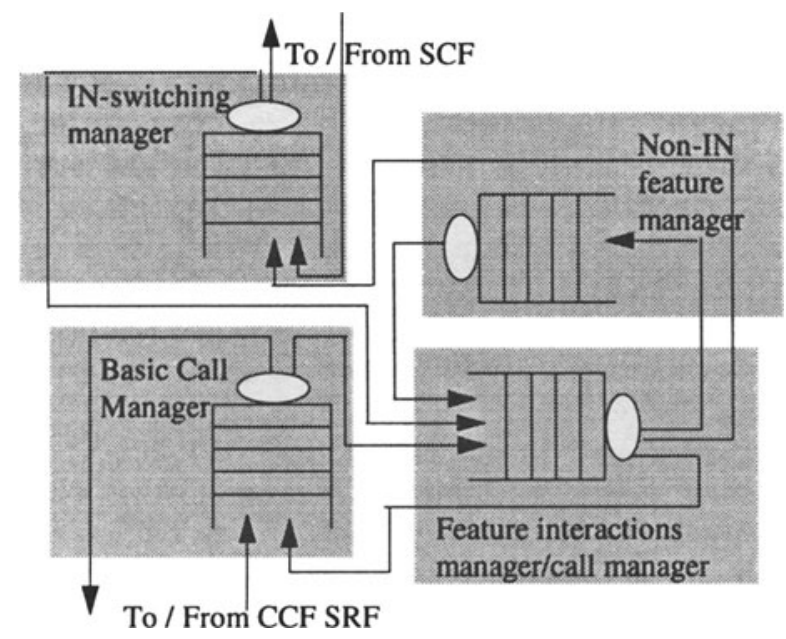

Figure 5 CCF/SSF Model.

CCF/SSF Model. The CCF/SSF Model of Figure 5 consists of the Basic Call Manager (BCM), the Feature Interactions Manager/Call Manager (FIM/CM), the Non-IN Feature Manager (NIFM), and the IN-Switching Manager (IN-SM).

The BCM detects basic call and connection control events that can lead to the invocation of IN service logic instances or should be reported to active IN service logic instances. The IN-SM interacts with the SCF in the course of providing IN service features to users. It detects IN call/connection processing events that should 


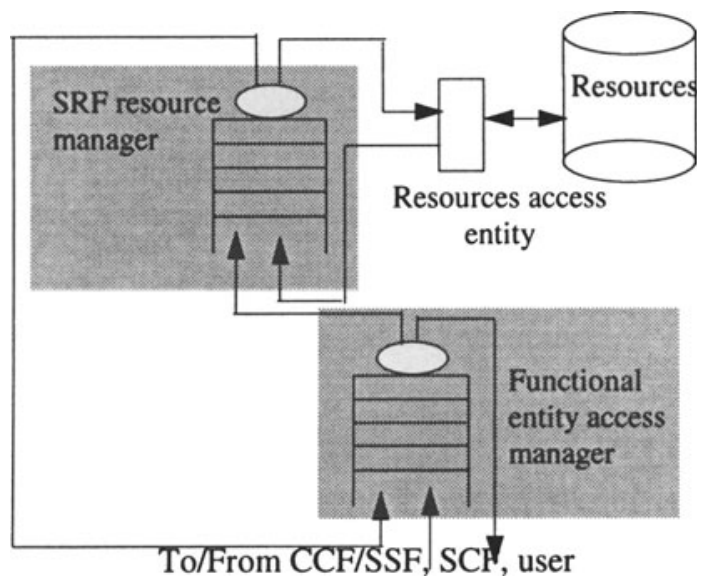

\section{Figure 6 SRF Model.}

be reported to active IN service logic instances. The FIM-CM provides mechanisms to support multiple concurrent instances of IN service logic instances and non-IN service logic instances on a single call. The NIFM executes the non-IN service logic instance.

SRF Model. The SRF model of Figure 6 consists of two components: the Functional Entity Access Manager (FEAM) which provides the necessary functionality to exchange information with other functional entities and the SRF Resource Manager SRF-RM which manages resources contained in the SRF.

SCF Model. The SCF model of Figure 7 consists of five sub-models and two libraries. These are the Functional Entity Access Manager (FEAM), the Service Logic Program Manager (SLPM), the Service Logic Execution Manager (SLEM), the Functional Routine Manager (FRM), the SCF Data Access Manager (SCFDAM), the Functional Entity Access Manager (FEAM) and the Service Logic Program (SLP) and Functional Routine (FR) Libraries. The FEAM provides the necessary functionality to exchange information with other functional entities and interacts with all the other service managers inside the SCF model. The SLPM manages the reception and distribution function of Service Logic Programs (SLPs) from other entities. The FRM is used for reception and distribution of functional routines to functional routine library via the library access entity. The SCF-DAM provides the functionality to storage, management and access of shared and persistent information in the SCF and the functionality to access remote information in SDFs. It interacts with the SLEM to provides these functionality. The SLEM handles and controls the total service logic execution and interacts with SCF-DAM. It also has access to SLP and FR libraries via the corresponding access entities in order to support the service logic execution.

SDF Model. The SDF model consists of two components the Functional Entity Access Manager (FEAM) which provides the necessary functionality to exchange information with other functional entities and the SDF Data Manager (SDF-DM) 


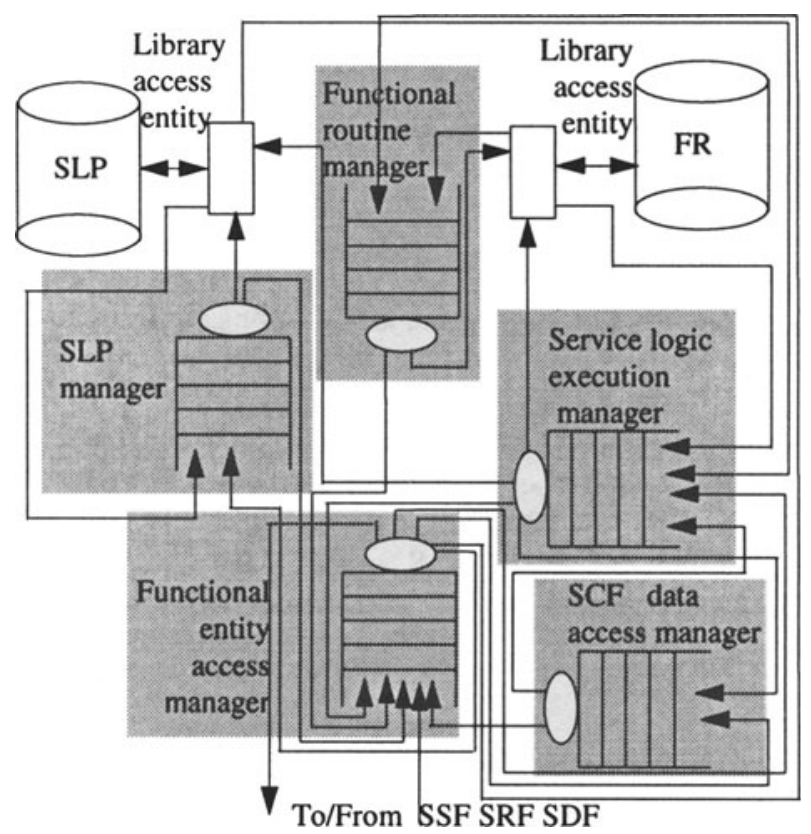

Figure 7 SCF Model.

which provides the necessary functionality to manage data contained in the SDF. It is modeled analogously to the SRF model (Figure 6).

\section{MESSAGE FLOWS AND WORKLOAD DEFINITION}

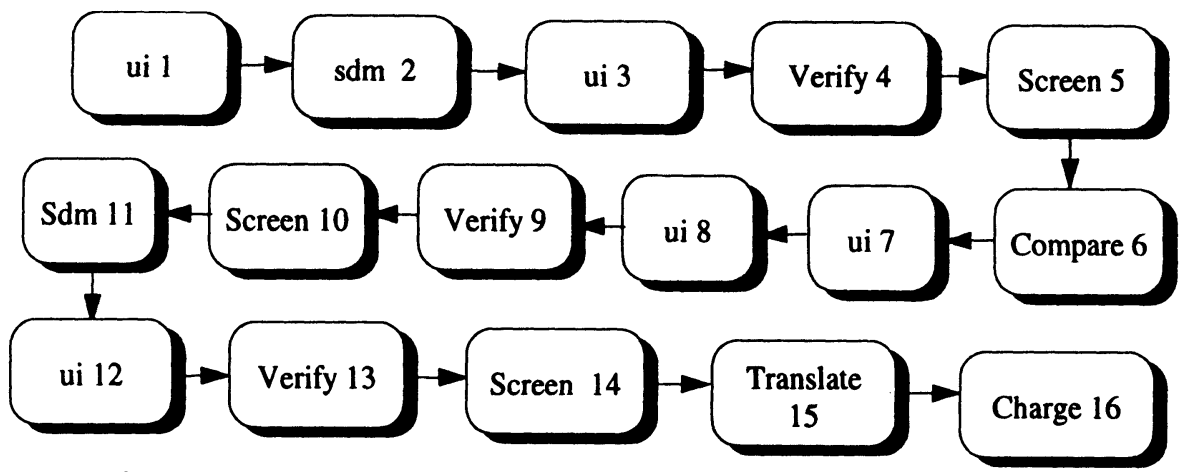

Figure 8 Global Service Logic for valid user's selections for B-VoD service.

The information flows among protocols and functional entities define the workload of the models presented so far. For simple signalling protocols that support a restricted number of well known services the information flows can be easily 
defined. The IN capability of the signalling network allows for services with very different characteristics in terms of protocol and functional entities interactions and the task of providing the detailed message flows becomes more difficult as this should be performed separately for each service.

Using the Global Functional Model (GFM) (ITU-T, Q.1203) we develop the Global Service Logic (GSL) of a typical B-VoD service from which the information flow among the functional entities of the system is derived. Initially the user is connected to a SRF in order to select a VS. Upon selection the connection is released and the user is connected to the VS for content selection. The process of the VS selection is shown by SIBs ui-1 to ui-7 in Figure 8, while the content selection by the remaining SIBs. In sequence, service control is passed to the Basic Call Process of the SSF which issues a B-ISUP IAM (Initial Address Message) to the VS to establish a connection between the user and the VS for video delivery. In the B-VoD service example the B-SCP orders the B-SSP to establish one connection between the user and the B-IP and two connections between the user and the VS, one for the controlling the video play and one for receiving video.

\section{PERFORMANCE RESULTS}

\subsection{System Parameters}

The models presented in Section 3 can be used to obtain performance results either by analysis or simulation. Analytical models are based on the aggregation and decomposition principle: Each protocol layer of a stack is studied with an individual model. The aggregation of individual results leads to the estimation of the system performance. To obtain analytical solutions the Poisson assumption is usually employed (see for example developments in (Bafutto, 1994), (Veeraraghavan), (Willmann, 1990), (Bafutto, 1992), (Bafutto, 1993), (Ghosal, 1995)). Along this line arrivals at each protocol are modeled as Poisson. In the following we use the M/G/1 model with feedback and non-preemptive service priorities (Simon, 1984), (Paterok, 1989). The simulation programs were run on a Sun 20 workstation with a Sun 4.1.4 OS. A total of 250 secs of system time has been simulated which corresponds to 25,000 calls for the maximum call rate. For lower call rates the simulated system time was in the order of 1,000 s. The total CPU time to obtain all results was about four hours.

Two types of services are considered in our performance evaluation study, that is a simple telephony service requiring no IN functionality and a typical IN-based BVoD service which makes use of the IN functions in the manner described in Section 4. Traffic scenarios consist of mixed traffic of the aforementioned services; i.e. $85 \%$ of telephony and $15 \%$ of $\mathrm{B}-\mathrm{VoD}$. The maximum traffic load considered is equal to $80 \%$ of the Busy Hour Call Attempts (BHCA) of one exchange measured in the Singapore national SS7 network (Lazar, 1994). In the analysis external arrivals are modeled as Poisson for each protocol model while in the simulation this 
assumption is only used for user initiated external call arrivals at the B-SSP (Bafutto, 1994), (Willmann, 1990), (Smith, 1994), (Lekkou). For the B-VoD service we assume movies with a mean length of 110 minutes (Ghafir, 1994). Users either watch the whole movie (i.e. duration time of the call equal to 110 minutes) or stop the movie 5 minutes after their selection. Therefore the B-VoD duration $(d)$ in the simulation follows a constant distribution with $d=110 \mathrm{~min}$ with probability $p_{l}=0.85$ and $d=5 \mathrm{~min}$ with probability $p_{2}=0.15$. The duration of telephony follows a mixture of two normal distributions on logarithmic time scale, as described below:

$$
F(t)=\beta \cdot F_{1}(t)+(1-\beta) F_{2}(t)
$$

Realistic values for the parameters of the distribution in (1) are given in (Bolotin, 1994): mean call duration $150 \mathrm{sec}, \beta=0.4, \mu_{1}=1.31, \sigma_{1}=0.33, \mu_{2}=2.11, \sigma_{2}=$ 0.5 .

Table 1 Process time $\mathrm{T}(\mathrm{ms})$, process priority ps

\begin{tabular}{lllllllll}
\hline \multicolumn{2}{c}{$S C F$} & & \multicolumn{3}{c}{$B I S U P$} & \multicolumn{4}{c}{$S S F / C C F$} \\
proc & $T(m s)$ & $p s$ & proc & $T(m s)$ & ps & proc & $T(m s)$ & $p s$ \\
\hline FEAM & 0.3 & 1 & SACF & 0.3 & 4 & BCM & 0.5 & 3 \\
SLEM & 1.5 & 2 & BCC & 1.0 & 2 & FIM & 1.0 & 2 \\
DAM & 0.5 & 5 & CC & 0.5 & 3 & INSM & 1.0 & 1 \\
SLP_L & 0.5 & 3 & AP & 0.5 & 1 & & & \\
FRL & 0.5 & 4 & & & & & & \\
\hline
\end{tabular}

Table 2 Process time $\mathrm{T}(\mathrm{ms})$, process priority ps

\begin{tabular}{lllllllll}
\hline \multicolumn{2}{c}{$S C F$} & & \multicolumn{2}{c}{$S R F$} & \multicolumn{4}{c}{$S D F$} \\
proc & $T(m s)$ & $p s$ & proc & $T(m s)$ & $p s$ & proc & $T(m s)$ & $p s$ \\
\hline Inc & 0.15 & 2 & FEAM & 0.3 & 2 & FEAM & 0.3 & 2 \\
Outg. & 0.25 & 1 & RM & 0.5 & 1 & DM & 0.5 & 1 \\
\hline
\end{tabular}

In both analytical model and simulation we assume constant service times. The service times per protocol process and the process priority within a functional 
entity or protocol appear in Tables 1 and 2. The priorities of Tables 1 and 2 are defined according to the following rules: The internal process with the higher utilization that is crossed by multiple itineraries is always allocated the higher priority. If there is no process with the above features two cases are distinguished: if there are more than one processes that accepts external messages the process with the lower utilization due to external arrivals is allocated the higher priority. If there is only one process that accepts external messages the process with the lower utilization is allocated the higher priority. The B-ISUP processing times were defined using as input the values of (Veeraraghavan, 1995) for the time a message type spends in the entire protocol. Taking into account the nature of the specific processes activated when a message type enters the protocol machine, the protocol type has been decomposed into the process time components of Tables 1 and 2 . The Q.2931, CCF/SSF, SCF, SRF and SDF processing times have been defined according to the functions of each process using input from (Veeraraghavan). The message transfer delay between different network elements has been calculated as the time required to transmit the mean number of cells, a Q.2931, B-ISUP and BINAP signalling message is translated into, in a $1.5 \mathrm{Mbps}$ signalling channel (Veeraraghavan, 1995). The mean message length is calculated for all involving signalling and IN protocols and it is equal to two ATM cells, including the overhead of the underlying transfer protocols. The inter-message delay of successive user or server generated messages is assumed to be $100 \mathrm{~ms}$.

The metric used to capture the performance of the signalling system is the mean delay per protocol layer, functional and physical entity as well as the end-to-end mean set up delay; i.e., the time between a set-up message is issued by a user to the time a video channel is established from the VS to the user for B-VoD, and to the time a connect message is received by the user for telephony. Two sets of results are presented. The first captures the performance of signalling protocols and IN functional entities. The second set of results presents delays per signalling and/or IN network element as well as end-to-end delays. Both alternatives regarding the location of the SRF location are considered.

\subsection{Protocol and functional entity delay}

In Figures 9 and 10 the B-SSP, B-IP and exchange Q2931 mean delay is shown. We see that the Q.2931 delay is almost the same in the exchange (Figure 10) for both alternatives, while in the B-SSP the delay is slightly higher for alternative B. The reason is that the Q.2931 protocol is also activated for the communication between the B-SSP and the B-IP.

In Figures 11 and 12 the B-SSP, and the exchange B-ISUP mean delay is shown. Since the number of messages handled by these protocols is the same for both alternatives, the B-ISUP delay is kept almost stable. 


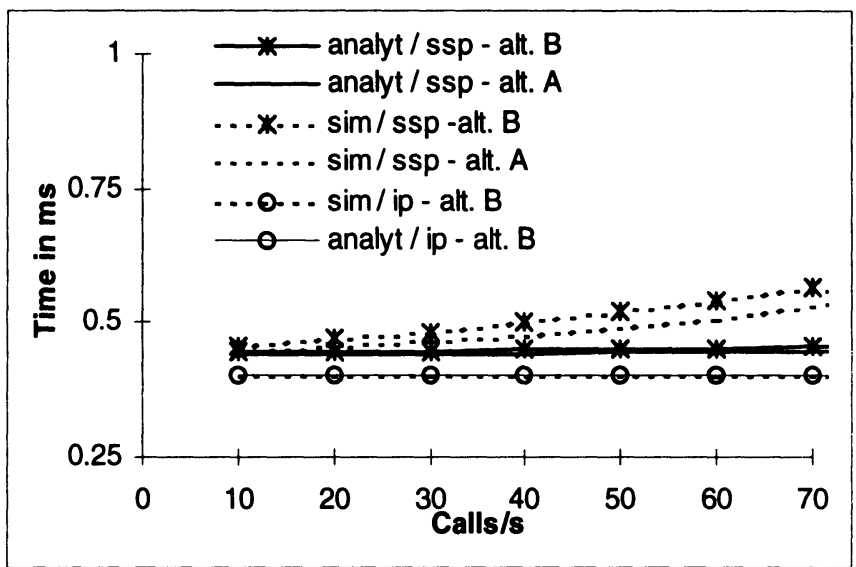

Figure 9 B-SSP / B-IP Q.2931 mean delay.

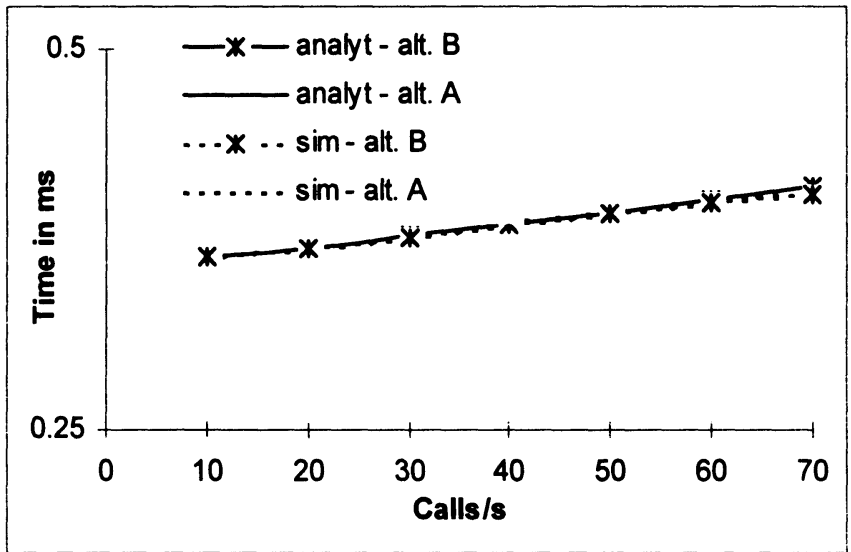

Figure 10 exchange Q.2931 mean delay.

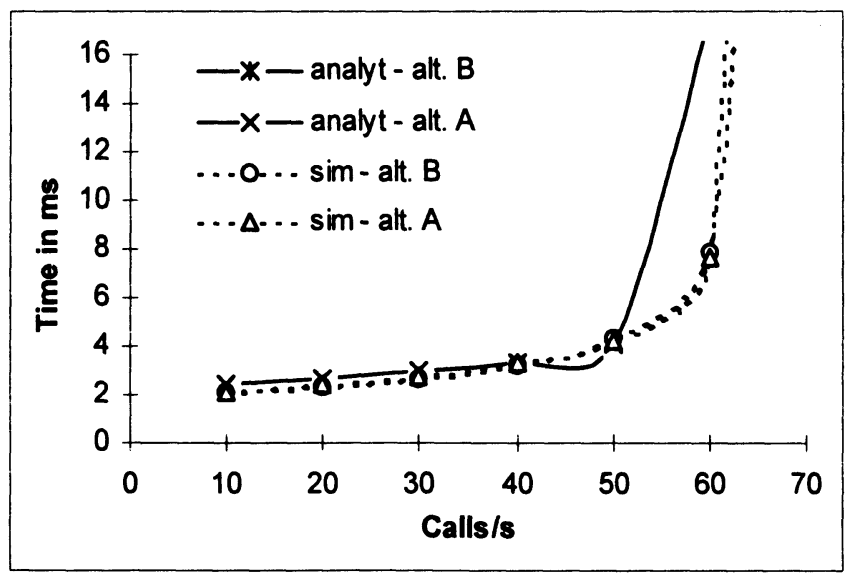

Figure 11 B-SSP B-ISUP mean delay. 


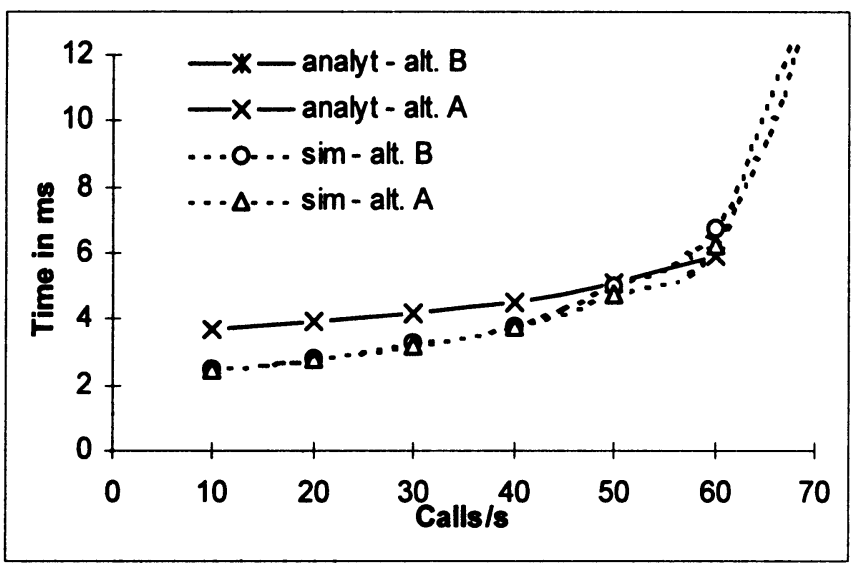

Figure 12 exchange B-ISUP mean delay.

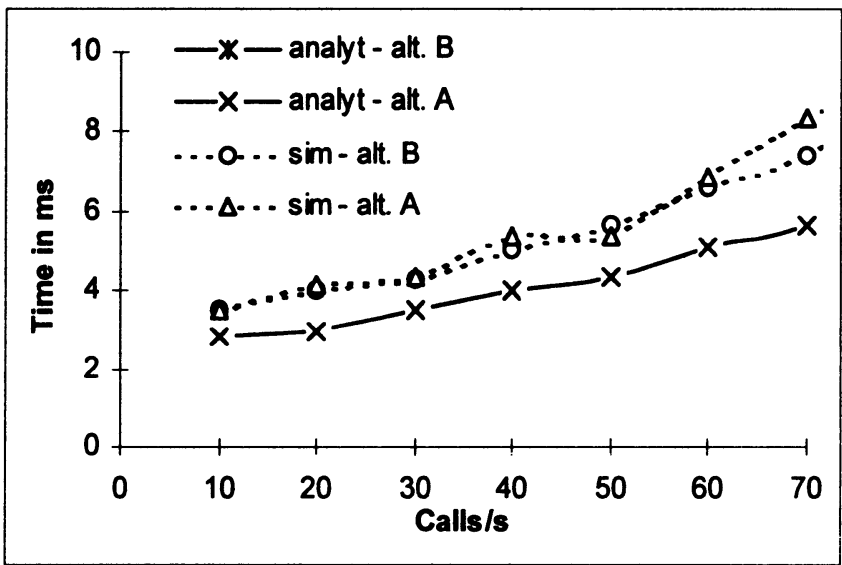

Figure 13 B-SCF mean delay.

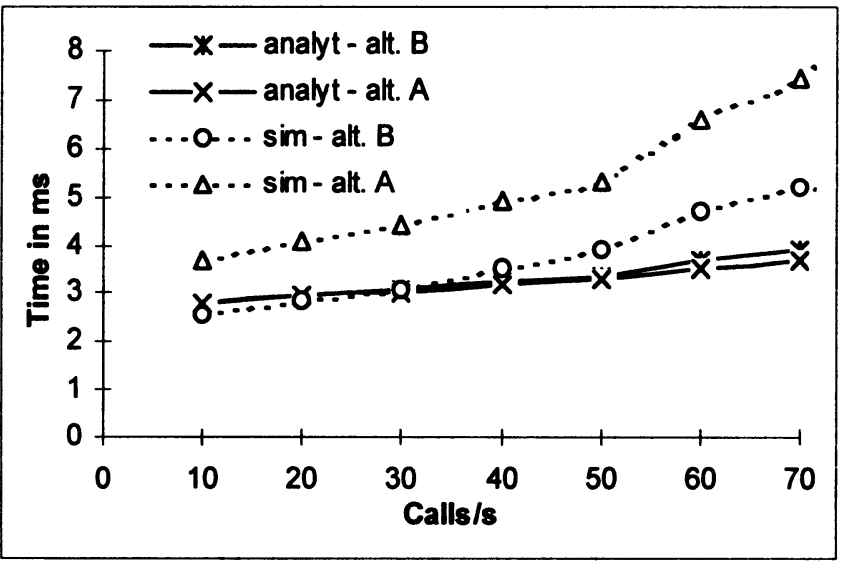

Figure 14 B-CCF/SSF mean delay. 
In Figure 13 the B-SCF mean delay is illustrated. Again the delay is the same in both alternatives, while the differences between the analytical and simulation results are due to the correlated nature of the IN messages arrived at the B-SCF. The Poisson assumption adopted in the analysis cannot accurately represent the IN message arrivals.

In Figure 14 the B-CCF/SSF mean delay is shown. Again the correlated nature of the IN messages arrived at the B-SSF justify the differences between simulation and analysis. We see that in simulation alternative B presents lower delay. This can be explained by consulting the message flow diagrams. In alternative $B$, messages entering the B-SSF present higher interarrival times while in alternative $A$ arrivals appear more bursty. These differences can not be derived from the analysis where we assume Poisson arrivals.

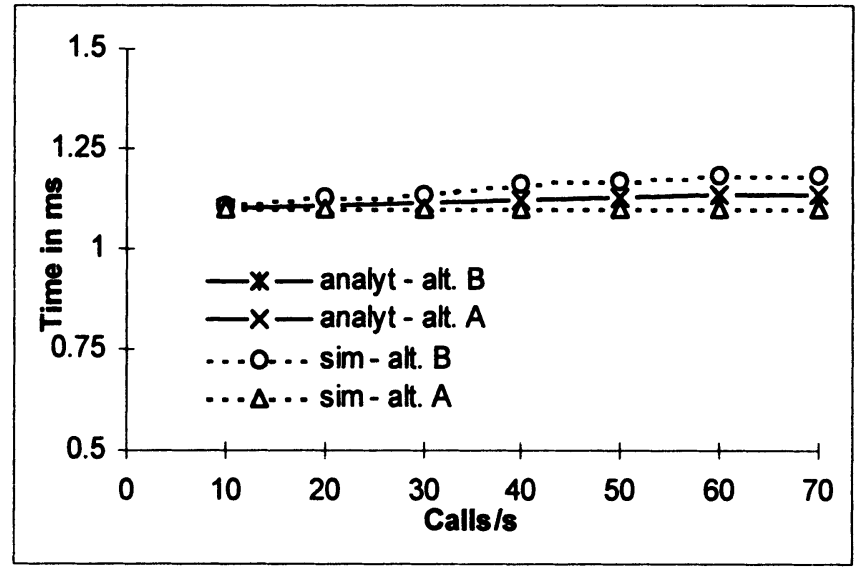

Figure 15 B-SRF mean delay.

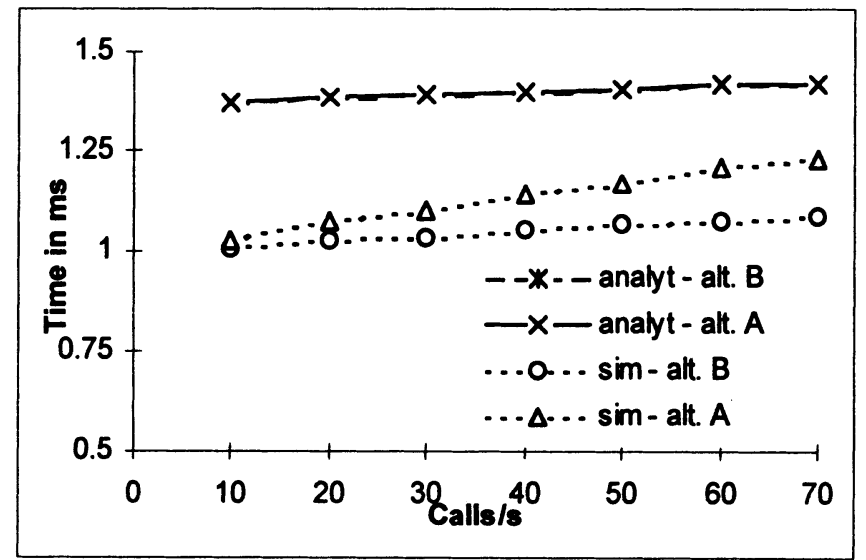

Figure 16 B-SDF mean delay. 
In Figure 15 the SRF mean delay is illustrated. We see that for both alternatives the delay is the same, since the load of the functional entity does not change. The same holds also for the SDF mean delay in Figure 16.

From the above figures it becomes apparent that the delay of protocols and functional entities do not vary with alternative A and B. Also we can see that the BISUP protocol determines the maximum number of calls that can be processed by the network. Therefore, while the SRF can serve more than 70 calls/s, the B-ISUP protocol of the B-SSP restricts this number to $60 \mathrm{calls} / \mathrm{s}$. This means that the same SRF can be used to serve more than one B-SSPs with acceptable performance which becomes possible only in alternative $B$.

\subsection{Physical entity and end-to-end delay}

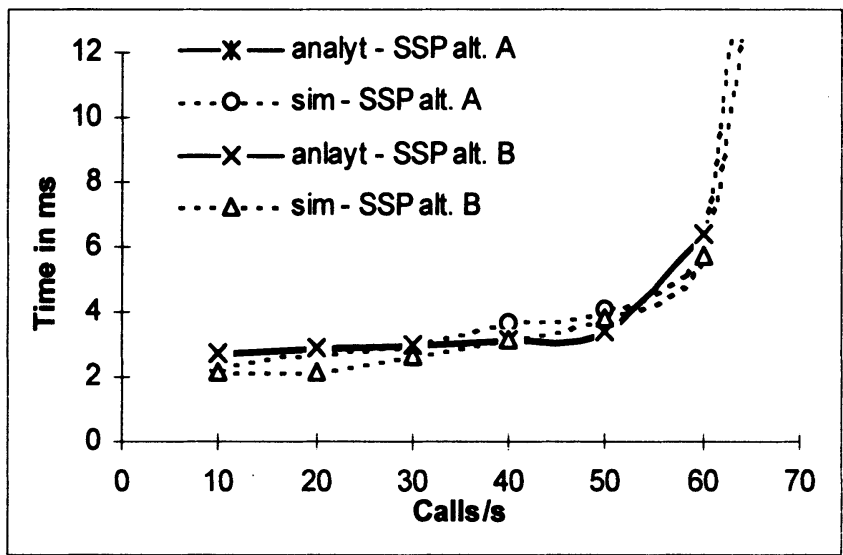

Figure 17 B-SSP mean delay.

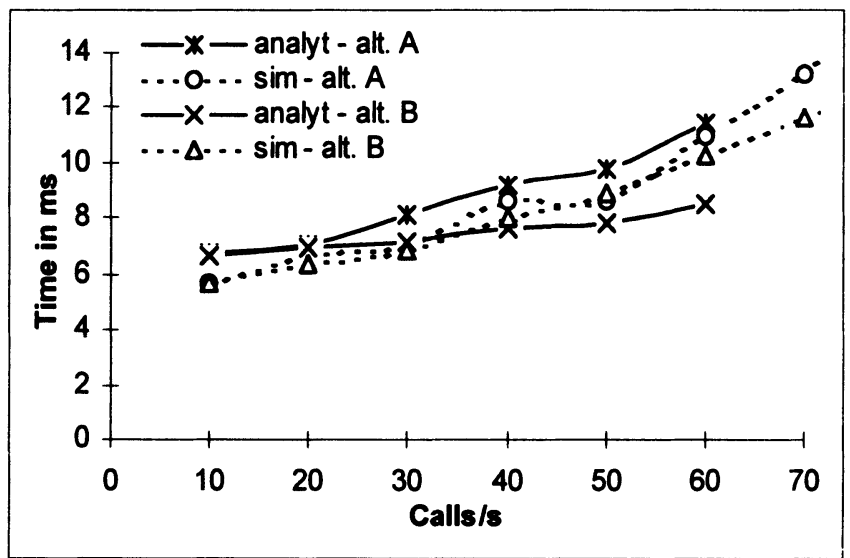

Figure 18 B-SCP mean delay. 


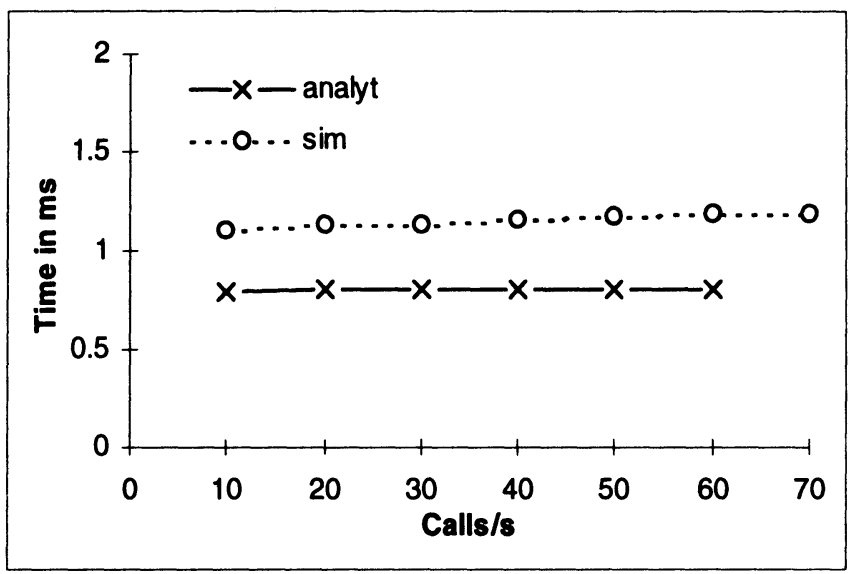

Figure 19 B-IP mean delay.

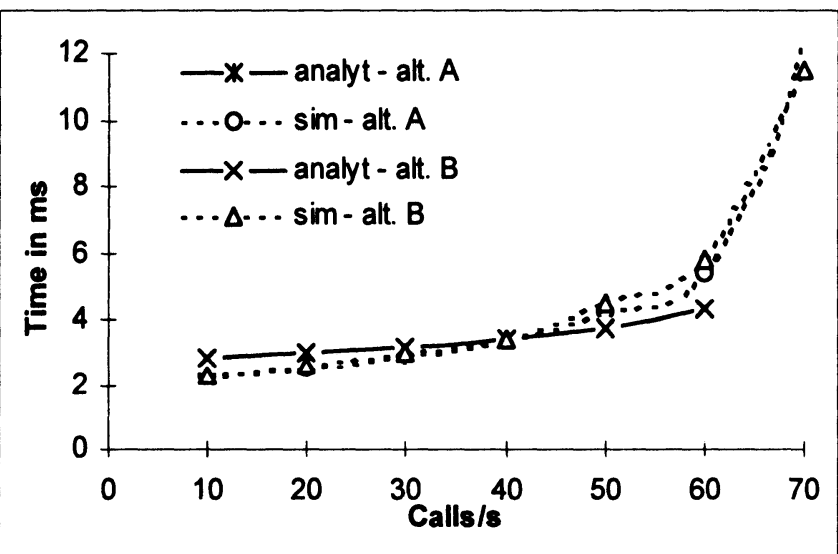

Figure 20 exchange mean delay.

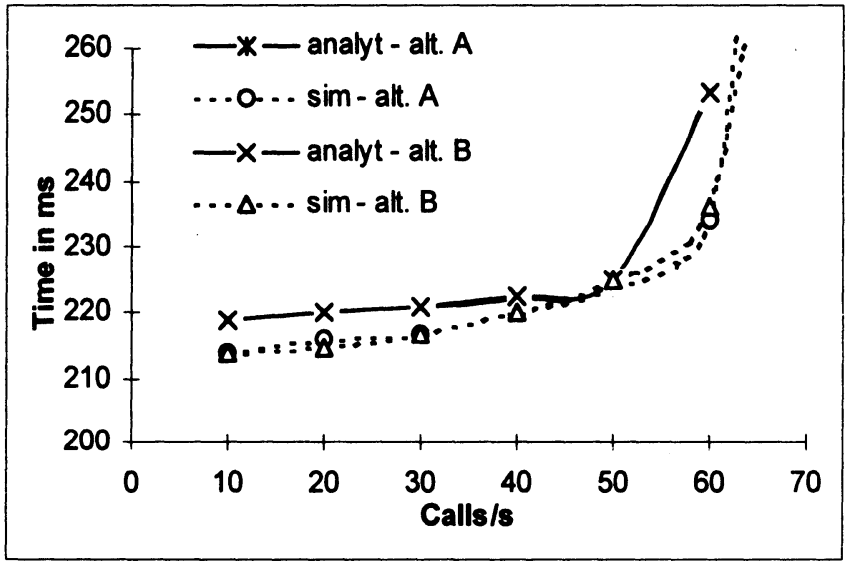

Figure 21 Telephony mean set-up delay. 


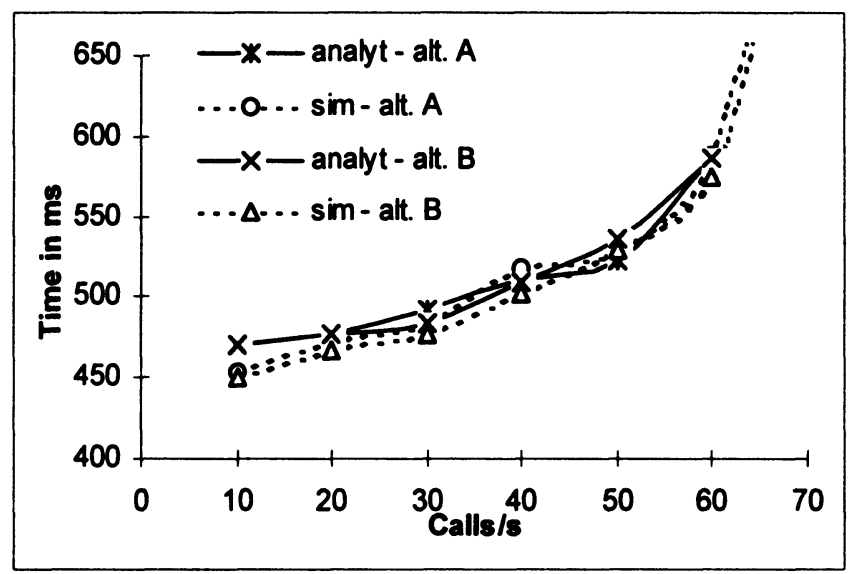

Figure 22 B-VoD mean set-up delay.

The remarks made for the functional entities also hold for the physical entities, where we can see that the delay is almost the same for both alternatives (see Figures 17-20). The B-IP mean delay is much lower than the B-SSP low, which further justifies the remark that the B-IP can support a considerably higher number of calls than the integrated B-SSP. We can also see that at the physical entity level the differences between results obtained by aggregating analytically calculated delay components and simulation become less intense and especially for the B-SSP which is located closer to the user and the exchange which does not process IN messages. Similar remarks can be made by consulting Figures 21 and 22 where we present the set-up delay for telephony and B-VoD respectively.

\subsection{B-IP Maximum number of supported calls}

In Figure 23 we plot the mean delay of a standalone B-IP corresponding to alternative B for different loads. As we have seen a B-SSP integrated or not can support up to 60 calls/s. Only 9 calls/s are B-VoD calls and are processed by the SRF. As shown in Figure 23 the B-IP can support up to $160 \mathrm{~B}-\mathrm{VoD}$ calls/s which means that about $16 \mathrm{~B}-\mathrm{SSP}$ can use the same B-IP with acceptable performance. It is interesting to notice that even in the case where the processing times of the B-IP functional entities and protocols increase 10 times (Tx10), the B-IP can still support more calls than a B-SSP. This is shown in Figure 24 where we find that the calls supported by the B-IP extends to $19 \mathrm{~B}-\mathrm{VoD}$ calls/s which is almost double than the corresponding number of the B-SSP. 


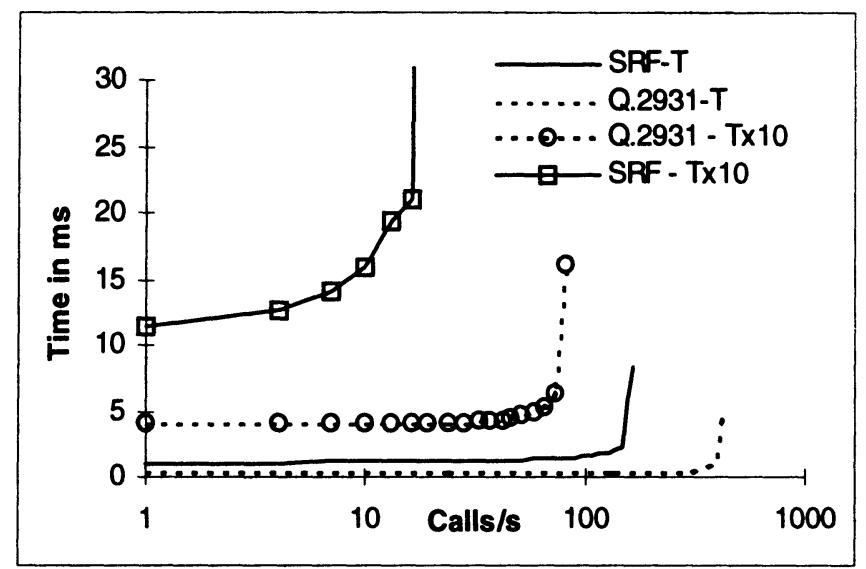

Figure 23 SRF, B-IP Q.2931 mean delay.

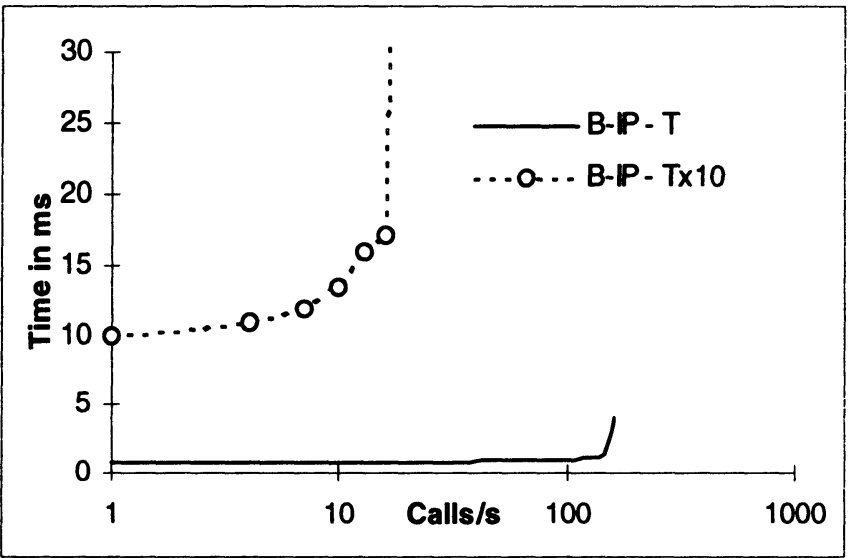

Figure 24 B-IP mean delay.

\section{CONCLUSIONS}

Several architectural issues of an IN/B-ISDN integrated system have been investigated in this paper from a performance viewpoint. To do so, a set of models for each functional entity and upper layer signalling protocols has been developed and information flows have been derived using the GSL methodology. The models are general enough to be used for other related performance studies. In our case the models have been used to capture the performance of all entities, functional or physical, involved in the establishment and release of an IN service call and to identify the most heavy loaded processes that constitute the system bottleneck. Special emphasis has been put on the location of the SRF for which two alternatives have been investigated. Our results have shown that for one B-SSP the performance of the system is almost stable independently of whether the SRF is 
embedded into an integrated and therefore more complex B-SSP or located in a standalone B-IP connected with a simpler B-SSP. Since the B-ISUP protocol inside the B-SSP is the one that determines the overall number of supported calls, the BIP alternative becomes more advantageous as a single standalone B-IP can serve multiple non integrated and therefore less complex B-SSPs. The authors are currently engaged in the development of a B-IP which will be part of an integrated IN/B-ISDN transnational european broadband network built to realise multimedia IN services.

\section{ACKNOWLEDGEMENT}

This work was partially funded by the European Union Advanced Communication Technologies and Services (ACTS) Project INSIGNIA (IN and B-ISDN Signalling Integration on ATM Platforms) (INSIGNIA). The opinions appearing in this paper are those of the authors and not necessarily of the other members of the project consortium. The authors wish to express their gratitude to all members of the consortium for fruitful technical discussions.

\section{REFERENCES}

Bafutto, M., Kuehn, P. J. and Willmann, G. (1994) Capacity and Performance Analysis of Signaling Networks in Multivendor Environments. IEEE J. Select. Areas Commun, Vol. 12, No. 3, 490-500.

Hussmann, H., Straten, G.v.d., Theimer, Th. and Totzke, J. (1995) An IN-based Implementation of Interactive Video Service. Proceedings of ICC'95, Seattle.

Lazar, A.A., Tseng, K.H., Lim, K.S. and Choe, W. (1994) A Scalable and Reusable Emulator for Evaluating the Performance of SS7 Networks. IEEE J. Select. Areas Commun., Vol. 12, No. 3.

Veeraraghavan, M., La Porta, T.F., and Lai, W.S. (1995) An Alternative Approach to Call/Connection Control in Broadband Switching Systems. IEEE Commun. Mag., 90-96.

Veeraraghavan, M., Choudhury, G., and Kshirsagar, M. ( ) Implementation and Analysis of PCC (Parallel Connection Control), submitted for presentation.

Willmann, G., Kuhn, P.J., (1990) Performance Modeling of Signaling System No.7. IEEE Commun. Mag., Vol. 28, No. 7, 44-56.

Bafutto, M., Kuhn, P.J. (1992) Capacity and Performance Analysis for Signaling Networks Supporting UPT. Proceedings of Eighth ITC Specialist Seminar on Universal Personal Telecommunication, Santa Margherita Ligure (Genova), Italy, Sess. VII, Paper 1, pp. 201-213.

Hou, X., Kalogeropoulos, N.D., Lekkou, M.E., Niemegeers, I.G., and Venieris, I.S. (1994) A Methodological Approach to B-ISDN Signaling Performance. Int.J. of Commun.Sys., Special Issue: Signaling Protocols and Services for Broadband ATM Networks, Vol. 7, No. 2, 97-111. 
Simon, B. (1984) Priority Queues with Feedback. J. ACM, Vol. 31, No. 1.

ITU-T, Rec. Q.2761-4, Broadband Integrated Services Digital Network User Part (BISUP), 1994.

ITU-T, Rec. Q.2931, Broadband Integrated Services Digital Network (B-ISDN). Digital Subscriber Signalling No.2 (DSS 2). User Network Interface Layer 3 Specification for Call/Connection Control, 1994.

ITU-T, Rec. Q1203, Intelligent Network Global Functional Plane Architecture, 1993.

ITU-T, Rec. Q1213, Global Functional Plane for Intelligent Network CS-1, 1993.

ITU-T, Rec. Q1214, Distributed Functional Plane for Intelligent Network CS-1, 1993.

ITU-T, Rec. Q1218, Interface Recommendations for Intelligent Network CS-1, 1993.

ITU-T, Rec. Q2100-30, B-ISDN Signalling ATM Adaptation Layer, 1994.

ITU-T, Rec. Q771-5, Signalling System No.7 - Transaction Capabilities (TCAP), 1993.

ITU-T, Rec. Q701, Functional description of the Message Transfer Part (MTP) of Signalling System No.7.

ITU-T, Rec. Q711-4, Signalling System No.7 - Signalling Connection Control Part (SCCP).

Smith, D. (1994) Effects of Feedback Delay on the Performance of the TransferControlled Procedure in Controlling CCS Network Overloads. IEEE J. Select. Areas Commun., Vol. 12, No.3.

Lekkou, M. and Venieris, I.S. A Workload Model for Performance Evaluation of Multimedia Signalling Systems. Accepted for publication in Computer Communications.

Bafutto, M. (1993) A Modelling Approach for the Intelligent Network Application Protocol. Proceedings of the ITC Regional Seminar on Teletraffic Challenges for Developing Countries, Brasilia, Brazil, 61-70.

Paterok, M. and Fisher, O. (1989) Feedback Queues with Preemption Distances Priorities. ACM Sigmetrics Performance Evaluation Review, Vol. 17, No. 1, 136-145.

La Porta, T.F. and Veeraraghavan, M. (1993) Evaluation of Broadband UNI Signaling Protocol Techniques. Journal of High Speed Networks, 2(3).

Ghafir, H. and Chadwich, H. (1994) Multimedia Servers - Design and Performance. Proceedings of Globecom '94, USA.

Bolotin, V. (1994) Modelling Call Holding Time Distributions for CCS Network Design and Performance Analysis. IEEE J. Select. Areas Commun., Vol. 12, No.3, 433-438.

INSIGNIA project description (URL:http://www.fokus.gmd.de /nthp/ insignia/ entry.html).

Wu, T.-H., Yoshikai, N. and Fujii, H. (1995) ATM Signalling Transport Network Architectures and Analysis. IEEE Commun. Mag.. 
Ghosal, D., Lakshman, T.V. and Huang, Y. (1995) Parallel Architectures for Processing High Speed Network Signalling Protocols. IEEE/ACM Trans. Netw., Vol. 3, No. 6.

\section{BIOGRAPHY}

George T. Kolyvas was born in Corfu, Greece, in 1970. He received the Dipl.-Ing. degree from the Electrical Engineering Department of the University of Patras, Greece in 1993. He is currently a Ph.D. candidate in the Electrical and Computer Engineering Department of the National Technical University of Athens (NTUA), Athens, Greece. Mr. Kolyvas is a research assistant in the Telecommunications Laboratory of NTUA performing research in the area of B-ISDN access networks, signalling, multimedia service design, Intelligent Network technology, performance evaluation, modeling and queueing theory. He has participated in several European Union projects and is currently involved in the INSIGNIA AC068 project. Mr. Kolyvas has received several national awards for the entire five years of his undergraduate study. He is a member of IEEE and the Technical Chamber of Greece.

Spyros E. Polykalas was born in Kefalonia, Greece, in 1971. He received the Dipl. -Ing. Degree from the Electrical Engineering Department of the University of Patras, Greece in 1994. Since 1994 he is a Ph.D. candidate in the Electrical and Computer Engineering Department of the National Technical University of Athens (NTUA), Athens, Greece and research associate of the Telecommunications Laboratory. His research interests are in the area of signalling, Intelligent Networks, Personal Communications, performance evaluation and modeling. He has participated in several European Union ACTS projects and is currently involved in the INSIGNIA AC068 project. He has received several national awards for the entire five years of his undergraduate study. Mr. Polykalas is a member of IEEE and the Technical Chamber of Greece.

Iakovos S. Venieris was born in Naxos, Greece. He received the Dipl. -Ing. degree from the University of Patras, Greece in 1988, and the Ph.D. degree from the National Technical University of Athens (NTUA), Athens, Greece, in 1990, all in electrical and computer engineering. From 1994 he is an Assistant Professor in the Electrical and Computer Engineering Department of NTUA. His research interests are in the fields of B-ISDN, signalling, resource scheduling and allocation for network management, modeling, performance evaluation and queueing theory. He has over sixty publications in the above areas. Dr. Venieris has been exposed to standardisation body work and has participated in several European Union and national projects dealing with B-ISDN protocols, Intelligent Networks, ATM switching and access techniques. Dr. Venieris is a member of IEEE and the Technical Chamber of Greece. 